\title{
GLIBERTY-GENERALIZED MODEL FOR SPECTRAL CHARACTERISTIC OF CONIFEROUS LEAVES AND BROAD-LEAVED LEAVES
}

\author{
Yunkai Guo, Guanxing An, Chao Feng, Qiong Xie, Jian Li, Fengsong Zhou \\ Changsha University of Science \& Technology, School of Traffic \& Transportation Engineering \\ Changsha, Hunan, China, 410004, guoyunkai226@163.com
}

KEY WORDS: The GLIBERTY model; leaf structure parameters; infinite layer; cell diameter hierarchical computing theory;

\begin{abstract}
:
The application of different leaf physical models will result in the low accuracy in the process of the vegetation information inversion of the expressway, as well as poor efficiency in the process. The GLIBERTY model attempts to simulate the leaf reflectance and transmittance by the use of hierarchical computing theory, through the analysis of the optical properties of the single cell and the introduction of monolayer cells and cells of infinite layer between the reflectance and transmittance mathematical relationship,based on the radiative transfer theory. The study established the completed GLIBERTY model calculation theory system after the impaction of leaf structure parameters . The results show that the GLIBERTY model can accurately simulate the spectrum information of broadleaf and needle even in the case of less input parameters.
\end{abstract}

\section{INTRODUCTION}

Since the International Conference proposed the concept of "environmental impact assessment", Chinese researchers conducted in-depth researches on the environmental impact assessment on high-grade roadside vegetation according to actual requirements. Currently, the relative advanced method is the inversion of vegetation physical and chemical parameters and construction of vegetation environmental assessment system by taking advantage of the quantitative remote sensing and taking the remote sensing image as basis. The two widely used vegetation models during research are statistical model and radiation transfer model, while the radiation transfer model is popular among many researchers for its stability and wide applicability ,just as Kuusk(Kuusk ,1991) mentioned. At present, the physical model relatively and widely used for the characteristics of broad leaved vegetation is PROSPECT model. Later researchers established a LIBERTY model based on the research on the structural characteristics of coniferous leaves to simulate the optical characteristics of coniferous leaves. However the species and spatial pattern of roadside vegetation are distributed as mixed broad-leaved and coniferous band distribution. The spatial distribution pattern results in that the premise of physical model application is to carry out corresponding classification to vegetation. This process greatly affects the inversion efficiency of vegetation information, and the accuracy of vegetation classification will greatly affect the accuracy of vegetation information acquired, which hampers physical models to play their advantages to an extreme; Meantime, the diversity of the application of physical models results in the complexity of input parameters and the difficulty of obtaining parameters. A generalized model GLIBERTY is established by setting unified parameters for above problems met in the application of models under the full consideration of macro characteristics and microscopic characteristics broad leaves and coniferous leaves. The research result shows that the generalized model has good applicability and can simulate the optical information of one single leaf with relevant parameters under the situation where the coniferous leaves and broad leaves are not distinguished .

\section{MODEL THEORY}

The interaction of solar radiation and a single leaf is achieved with the chemical substances inside the leaf and the leaf structure. The pigment, water, cellulose, lignin and other components inside the leaf and corresponding concentration difference of them constitutes the absorption characteristic of the leaf among the optical characteristics and is the major cause of the reflectivity characteristic of visible light; Leaves shows high reflectivity in the near infrared band, which is mainly caused by the multi-reflection of lights on cell walls and air gaps. The research shows that the more the cell layers, the higher the spectral reflectance and the more obvious the anisotropies of cell shape and composition. Although there is shape difference between broad leaves and coniferous leaves, there is a big similarity in cell dimension between them and the mesophyll cells is spherical.

The arrangement and structure of mesophyll cells are the main difference between broad leaves and coniferous leaves. The mesophyll cells of broad-leaved leaves are arranged symmetrically along the axial and neatly on a plane, while coniferous leaves are arranged nearly centrically and circlewise; there is a obvious air interlayer between mesophyll cells of broad leaves and this interlay causes discontinuous refraction; while there is no obvious layered structure between mesophyll cells of coniferous leaves and the mesophyll cells like a pile of stacked cells ( Fig. 1).
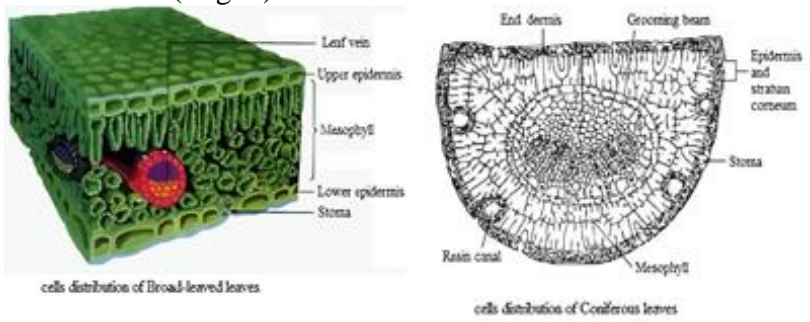

Figure 1. Arrangement of cells 


\subsection{Optical characteristics of one single leaf cell}

Terence P. Dawson(Terence P. Dawson ,1998) applied Optical Properties of Powders theory to get the basic equations of leave. Assuming that the cells in leaves are in standard spherical particles and the scattering on their surface complies with Lambert's cosine law (when the radiation brightness and light brightness of radiation sources in each direction are equal to that in each direction on the surface hemisphere, the radiant intensity changing along with the observation direction and angle between surface sources follows the cosine law) and assuming that the average reflection coefficient of air incoming cells is me, the average reflection coefficient transmitted from cells inside is mi.

For a standard sphere, the angle of refracted ray out from the sphere is always equal to that of ray into the sphere. The inner reflection coefficient and outer reflection coefficient of cells are determined according to Frensel equation. To speed up calculation, the calculation of the two reflection coefficients are done with transmission coefficient indirectly. The calculation equation for inverse exported reflectivity of interface transmittance without absorption action is:

$$
\begin{aligned}
\mathrm{m}_{\mathrm{e}} & =1-\mathrm{t}_{12} \\
\mathrm{~m}_{\mathrm{i}} & =1-\mathrm{t}_{21}
\end{aligned}
$$

Among which medium 1 represents air arrange cells and medium 2 represents the environment inside cells and that the refractive index is $n$.

$\mathrm{t}_{\mathrm{av}}(\alpha, 1, \mathrm{n})$ represents the interface transmittance whose integration is achieved by Judd in 1942 firstly. After that, parts researchers conduced complete calculation and research to it. When $\alpha$ is 0 , it represents vertical incidence; when $\alpha$ is 90 , it represents incidence of natural reflected light; when $\alpha$ is a arbitrary value, there is no dominant expression. $t_{a v}(\alpha, 1, n)$ represents the transmittance of light penetrating cells with reflective index of 1 ; similarly, $t_{a v}(\alpha, 1, n)$ represents the transmittance of light penetrating cells with reflective index of $\mathrm{n}$. The relationship between the two is:

$$
\mathrm{t}_{\mathrm{av}}(\alpha, \mathrm{n}, 1)=\mathrm{n}^{-2} \mathrm{t}_{\mathrm{av}}(\alpha, 1, \mathrm{n})
$$

Thus the inner reflection coefficient and outer reflection coefficient can be calculated by:

$$
\begin{gathered}
\mathrm{m}_{\mathrm{e}}=1-\mathrm{t}_{\mathrm{av}}(90,1, \mathrm{n}) \\
\mathrm{m}_{\mathrm{i}}=1-\frac{\mathrm{t}_{\mathrm{av}}(90,1, \mathrm{n})}{\mathrm{n}^{2}}
\end{gathered}
$$

The absorption coefficient of leaf cells is $\mathrm{K}$ (can be calculated according to their composition and concentration of compositions for the influence from chemical compositions of leaves). The radiant quantity entering cells at solid angle of $\mathrm{d} \theta$ in the direction of $\theta$ should be

$$
\frac{1}{\mathrm{pi}} \mathrm{e}^{-\mathrm{kd} \cos \theta} \cos \theta * 2 \mathrm{pisin} \theta \mathrm{d} \theta
$$

The total radiant quantity after one penetration for a interface $\mathrm{M}$ is:

$$
\mathrm{M}=2 \int_{0}^{\mathrm{pi} / 2} \mathrm{e}^{-\mathrm{kd} \cos \theta} \cos \theta \sin \theta \mathrm{d} \theta
$$

$$
\mathrm{M}=2\left[1-(\mathrm{kd}+1) \mathrm{e}^{-\mathrm{kd}}\right] /(\mathrm{kd})^{2}
$$

Because mi represents the average inner reflection coefficient, the initial radiant quantity from cells is $\mathrm{M}^{*} \mathrm{~m}_{\mathrm{i}}$. Assuming that the cell surfaces have the same radiation properties, for geometrical progression with total radiant quantity from of $\mathrm{M}^{*} \mathrm{~m}_{\mathrm{i}}$ after infinite inter mutual reflection, the total transmission quantity from cells is

$$
\tau=\frac{\mathrm{M}\left(1-\mathrm{m}_{\mathrm{i}}\right)}{1-\mathrm{Mm} \mathrm{m}_{\mathrm{i}}}
$$

Each spherical cell has the same optical properties, namely, the same transmittance $\tau$ (Judd, D. B,1942). When one beam of light penetrates cells, a part of radiation quantity is reflected with the reflectivity of me, and the rest of it enters parts of cells with radiation quantity of $\left(1-\mathrm{m}_{\mathrm{e}}\right)$ and this part of light emits from cells with radiation quantity of $\left(1-m_{e}\right) \tau$ after numerous internal reflections

\subsection{Reflectivity correction of infinite layer cells}

When a large number of leaves are stacked to form a certain thickness, it can be deemed that it is overlaid by infinite layer cells. The reflectivity is assumed to be $R$, and we believe that the reflection will not be changed if we place another layer of leaf on this leaf stack and the leaf cells are assumed to be arranged horizontally at this time. Terence P. Dawson applied Optical Properties of Powders theory to get the basic equations of leave. Assuming that the cells in leaves are in standard spherical particles and the scattering on their surface complies with Lambert's cosine law and assuming that the average reflection coefficient of air incoming cells is $m_{e}$, the average reflection coefficient transmitted from cells inside is $\mathrm{m}_{\mathrm{i}}$. The reflection coefficient can be Calculated by Stern 's transmittance calculation theory.

Just as Terence P. Dawson getting the basic infinite reflectivity function, assuming that all mesophyll cells are in the cell layer and they are superficial cells with layered structure, when the number of leaves is high, the uppermost layer of cells can be deemed as the infinite layer cell enveloping with total reflectivity of $\mathrm{R}$ and the reflectivity of the air layer on the uppermost layer of cells is $\mathrm{R}$ too in theory. we can define three variables: $\mathrm{x}_{\mathrm{u}}$ : upward radiation factor from cells; $\mathrm{x}_{\mathrm{a}}$ : lateral radiation factor from cells; $\mathrm{x}_{\mathrm{d}}$ : downward radiation factor from cells. The three factors transmit to upper cells, adjacent cells and lower cells respectively. Ideally, $\mathrm{x}_{\mathrm{u}}$ radiation factor transmitting upwards and $\mathrm{x}_{\mathrm{d}}$ radiation factor transmitting downwards just scatter among a solid angle close to $2 \pi$. Decomposing the lateral radiation factor $\mathrm{x}_{\mathrm{a}}$ into a upward factor and a downward factor without loss can simplify the scattering radiation. To facilitate calculation, we can redefine a probability parameter $X$ to represent the total radiation of this part transmitting from cells towards, and the $\mathrm{X}$ can be defined as:

$$
\mathrm{X}=x_{u}+\left(\tau x_{a}\right)+x_{u}\left(\tau x_{a}\right)^{2}+x_{u}\left(\tau x_{a}\right)^{3}+\cdots
$$

Can be obtained with summation formula about geometric sequence:

$$
\mathrm{X}=\frac{x_{u}}{1-\tau x_{u}}
$$

Simplified as: 
Under normal circumstances, the absorption coefficient is low, so, : $\mathrm{Kd}<1$ and $\mathrm{x}_{\mathrm{u}}$ is approximately equal to $\mathrm{x}_{\mathrm{d}}$. Theoretically there is an equation: $x_{u}+{ }_{x a}+x_{d}=1$, so the result of $X$ can be simplified as:

$$
\mathrm{X}=\frac{x_{u}}{1-\left(1-2 x_{u}\right) \tau}
$$

Parameter $\mathrm{X}$ is important which characterizes the reflection function or scattering efficiency of cell air interlayers. The radiation of cells will more close to the real situation through addition of radiation quantity $\mathrm{X}$ from the lower space to the upper space.

In the process of interaction between monolayer cells and infinite layer cells, the total reflectivity $\mathrm{R}$ includes the results of the first reflection of incident ray, the second, the third and infinite times of reflection effect of incident ray after transmission (Fig. 2).

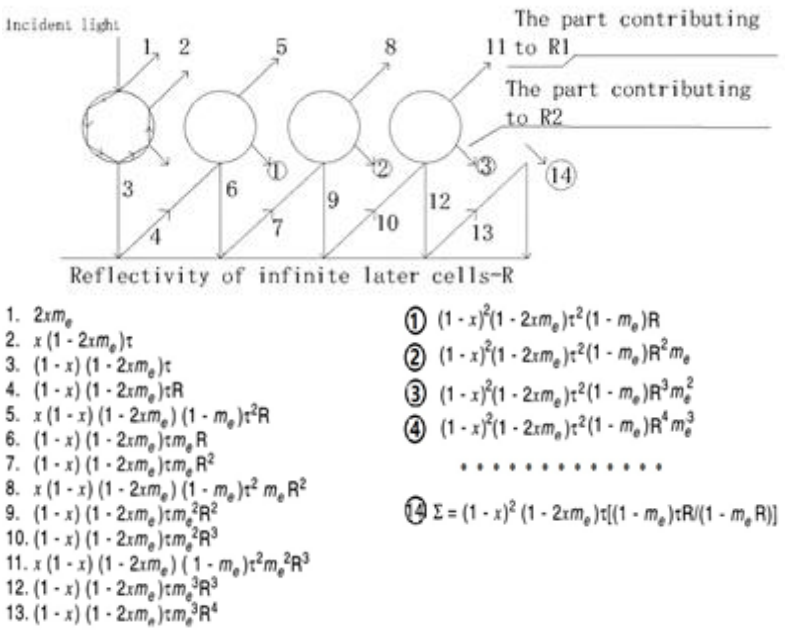

Figure 2. Interaction between monolayer cells and infinite layer cells

During the first reflection, the reflection factor me is limited in the $2 \pi$. spherical solid angle, there is no $x_{d}$, but there is a equation: $x_{d}=0, x_{u}$ is two times the upward radiation quantity from cells, so the radiation quantity in the first reflection is a part of the total reflectivity $\mathrm{R}$. Meantime, the radiation quantity transmitted into cells is $1-2 \mathrm{xm}_{\mathrm{e}}$. The part is divided into two parts after infinite reflection in cells: one part transmits upwards again and forms a part of total reflectivity $\mathrm{R}$ with radiation quantity of $x\left(1-2 \mathrm{xm}_{\mathrm{e}}\right)$ and the other part transmits downwards to the infinite cell layer with radiation quantity of $(1-\mathrm{x})(1-$ $2 \mathrm{xm}_{\mathrm{e}}$ ) . Because the total reflectivity of lower infinite layer cells is $\mathrm{R}$, some radiation transmits to the upper monolayer cells after reflection through the lower infinite cells and the some radiation quantities transmits out of cells and forms a part of the total reflectivity $R$. After the infinite timers of interaction between monolayer cells and infinite layer cells, the part of radiation transmitting from leaves and contributing to the total reflectivity $\mathrm{R}$ is:

$$
\begin{gathered}
2 \mathrm{x} m_{\theta}+x\left(1-2 x m_{\theta}\right) \tau+x(1-x)\left(1-2 x m_{\theta}\right) \tau^{2} R+ \\
x(1-x)\left(1-2 x m_{\theta}\right) \tau^{2} m_{\theta} R^{2}+ \\
x(1-x)\left(1-2 x m_{\theta}\right) \tau^{2} m_{\theta}^{2} R^{3}+\cdots
\end{gathered}
$$

The summation to it with geometric progression can be done as follows:

$$
\begin{gathered}
\mathrm{R} 1=2 \mathrm{x} m_{e}+x\left(1-2 x m_{e}\right) \tau+ \\
\underline{x(1-x)\left(1-2 x m_{e}\right)\left(1-m_{e}\right) \tau^{2} R}
\end{gathered}
$$

The structural mechanism of the infinite reflection as been validated in detail in Fig. 2. In addition to the part of radiation contributing to the total reflectivity $\mathrm{R}$ during the infinite process of action of the initial part, the second infinite reflection, the third infinite reflection and many times of reflection after them that transmit downwards from cells are added in the calculation. This part of radiation is the residual part of the above formula. For the radiation reflecting upwards from the subject and entering cells for the thirst time, a part of radiation transmits upwards through cells directly and form a part of formula (14), and the other part transmitting downwards and entering the air interlayers for the next infinite radiation with radiation quantity of:

$$
(1-\mathrm{x})^{2}\left(1-2 x m_{\Theta}\right) \tau\left(1-m_{e}\right) \tau R /\left(1-m_{\Theta} R\right)
$$

This part of radiation is divided into two parts again when reflecting into cells for the second time: one part conducts infinite reflection in cells and contributes to the total reflectivity and the other parts reflects into cell layer gaps again for the next reflection. The radiation quantity of the part contributing to the total reflectivity $\mathrm{R}$ is:

$$
\mathrm{x}(1-x)^{2}\left(1-2 x m_{e}\right) \tau\left[\left(1-2 x m_{e}\right) \tau R /\left(1-m_{e} R\right)\right]^{2}
$$

Accordingly, the radiation quantity of the part entering the cell layer gaps for next reflection is:

$$
(1-x)^{3}\left(1-2 x m_{e}\right) \tau\left[\left(1-m_{e}\right) \tau R /\left(1-m_{e} R\right)\right]^{3}
$$

The radiation quantity from monolayer cells actually can be determined with summation limit through such continuous reflection and transmission process, namely:

$$
\begin{gathered}
\frac{x(1-x)\left(1-2 x m_{e}\right)\left(1-m_{e}\right) \tau^{2} R}{1-m_{e} R}+ \\
x(1-\mathrm{x})^{2}\left(1-2 x m_{e}\right) \tau\left[\frac{\left(1-m_{e}\right) \tau R}{1-m_{e} R}\right]^{2}+x(1-x)^{3}(1- \\
\left.2 x m_{e}\right) \tau\left[\frac{\left(1-m_{e}\right) \tau R}{1-m_{e} R}\right]^{3}+\cdots
\end{gathered}
$$

Similarly, the method of summation formula about geometric sequence can be used to obtain the eventual formation of R2, namely:

$$
\mathrm{R} 2=\frac{x(1-x)\left(1-m_{\mathbb{L}}\right)\left(1-2 x m_{\underline{m}}\right) \tau^{2} R}{\left(1-m_{\mathbb{L}} R\right)-(1-x)\left(1-m_{\mathbb{L}}\right) \tau R}
$$

Thus, the total reflectivity of infinite cell layer can be deemed to be the sum of $\mathrm{R} 1$ and $\mathrm{R} 2$ :

$$
\begin{aligned}
\mathrm{R}=2 \mathrm{x} m_{e}+x\left(1-2 x m_{\varepsilon}\right) \tau+\frac{x(1-x)\left(1-2 x m_{e}\right)\left(1-m_{e}\right) \tau^{2} R}{1-m_{e} R}+ \\
\frac{x(1-x)\left(1-m_{e}\right)\left(1-2 x m_{e}\right) \tau^{2} R}{\left(1-m_{e} R\right)-(1-x)\left(1-m_{e}\right) \tau R}
\end{aligned}
$$

The equation(20) of $\mathrm{R}$ is a typical cubic equation that can be solved correctly with Newton- Laphson iteration. The initial 
value for calculation can be selected from a actually determined values, and can also deemed to be the solution of quadratic equation when $\mathrm{R}$ is equal to $\mathrm{R} 1$ for iteration. However, in iteration, it is required to change the equation to a standard cubic equation(Terence, P. D,1998), otherwise, the iteration error for multiplicity of solutions of the equation under limited conditions cannot be eliminated.

\subsection{Optical characteristics of cells of finite layer leaves}

The total reflectivity $\mathrm{R}$ obtained in 2.2 actually represents the reflectivity of cells of infinite layer coniferous leaves. Although the result contributes to the contractive analysis with the actual measured data in field, the spectral information of single coniferous leaves and even the canopy spectrum of the whole plant are required during the actual application of quantitative remote sensing. The accurate determination and research on coniferous spectrum in the laboratory shows that the backscattering of monolayer cells without lower materials will contribute to the total reflectivity. According to this theory, the reflectivity of monolayer cells is:

$$
\mathrm{R}=2 \mathrm{x} m_{e}+x\left(1-2 x m_{e}\right) \tau
$$

In Benford's research, the bidirectional reflectance of some material with a certain thickness for the same reflection and transmission properties changes along with the change of thickness and is a dependent variable with thickness as a independent variable. Therefore, the reflectivity of cell layers $\mathrm{R}$ is changeable within the scope of limited thickness. When the reflectivity $\mathrm{R}$ is stable, it means that the cell layers can be deemed as infinite layers and the relationship between the reflectivity and transitivity of infinite layers and finite layers can be deduced.

$$
R_{\infty}=R+\frac{R_{\infty} T^{2}}{1-R R_{\infty}}
$$

The formula connects the reflectivity of infinite layer and that of monolayer. Meantime, the calculation method for the infinite layer reflectivity has been deduced in the basic principle in the formula and there is calculation method for monolayer reflectivity. The transmittance $\mathrm{T}$ of monolayer cells can be obtained by simplifying the formula:

$$
\mathrm{T}=\sqrt{\frac{\left(R_{\infty}-R\right)\left(1-R R_{\infty}\right)}{R_{\infty}}}
$$

Different from the theory of continuous calculation of thickness in the physical model for coniferous leaves, optical sectioning calculation theory is introduced into a model to establish a model for leaves with clear structure and layering in this text. Relevant theories about the description and calculation of the physical process of light rays penetrating a series of plates for reflection and transmission are already in existence. LB TUCKERMAN organized theories proposed by researchers and proposed a complete calculation theory system has been given. Assuming that there is a light transmission model composed by $\mathrm{N}$ layers of plates and each layer has given reflection $\mathrm{R}$ and transmittance $\mathrm{T}$, the light intensity transmitting into this optical system will not change regardless of the incident angle of the light rays, the problem at this point is how to obtain the reflectivity $R_{N}$ and the transmittance $T_{N}$ of this system. From the physical characteristics of light, the two parameters must be parts of the function of reflectivity $\mathrm{R}$ and transmittance $\mathrm{T}$ of monolayer plates. Assuming that the system composed by the $\mathrm{N}$ layers of plates consists of $(m+n)$ two parts and the light transmits on the layer $\mathrm{m}$ plate firstly, the light intensity $r_{m}$ represent the light intensity transmitting back from the first part of plate and $t_{m}$ represents the light intensity transmitting out from first part of plate. For the next layer $n$ plate, the light intensity of $r_{n}$ will be reflected and that of $t_{n}$ will be transmitted. For plates consisting of two parts in whole, after infinite times of reflection and transmission, the reflected light intensity of the whole plate system is:

$$
r_{m+n}=r_{m}+t_{m}^{2} r_{n}+t_{m}^{2} r_{m} r_{n}^{2}+\cdots+t_{m}^{2} r_{n}\left(r_{m} r_{n}\right)^{k-1}+\cdots
$$

This theoretical process will not reduce the accuracy for the difference of assumed number of plates. If the plate is a monolayer plate, the applicability of the theoretical process can be demonstrated directly. The formula can be simplified as:

$$
r_{m+n}=r_{m}+\frac{t_{m}^{2} r_{n}}{1-r_{m} r_{n}}
$$

Similarly, the expression of $\mathrm{t}_{\mathrm{m}+\mathrm{n}}$ can be obtained:

$$
t_{m+n}=t_{m} t_{n} /\left(1-r_{m} r_{n}\right)
$$

At this time, when $m=1+n$, a recursion formula about $n$ can be obtained and then the reflectivity $r_{n}$ and transmittance $t_{n}$ of the system can be obtained.

L. B. TUCKERMAN simplified and resolved the above formulas and obtained a final simplified formula:

$$
\frac{t_{n}}{c-c^{-1}}=\frac{r_{n}}{d^{n}-d^{-n}}=\frac{1}{c d^{n}-c^{-1} d^{-n}}
$$

$\mathrm{r}$ and $\mathrm{t}$ represent the reflectivity and transmittance of a monolayer plate respectively and $\mathrm{n}$ represents a structural parameter;

The parameters are calculated as follows:

$$
\begin{gathered}
\mathrm{p}=\frac{1+t^{2}-r^{2}}{2} \\
\mathrm{q}=\sqrt{p^{2}-t^{2}} \\
\mathrm{c}=\frac{1-p+q}{r} \\
\mathrm{~d}=\frac{p+q}{t}
\end{gathered}
$$

\section{SETTING OF MODEL PARAMETERS}

Because the generalized model uses uniform calculation parameters without tell between coniferous leaves and broad leaves and them, relevant physical and chemical parameters about the two leaves must be supplemented during calculation. An important parameter among chemical parameters of leaves is the absorption coefficient $\mathrm{K}$, not matter whether the leaves are coniferous leaves or broad leaves, which represents the basic absorption action of biochemical substances of leaves to light waves under different wave band. In the mode for coniferous leaves, the absorption coefficient is regarded as the linear relation between chlorophyll and nitrogen content, while in actual application, describing the relationship with the abruption coefficient with pigment, dry matters, equivalent water thickness and other parameters is more important, so this model still uses this liner relation to represents the absorption coefficient, and the calculation formula of it is: 


$$
\mathrm{k}=\sum \frac{c(i) * k(i)}{N}
$$

Among which $\mathrm{C}(\mathrm{i})$ represents the concentration of a chemical substance; $\mathrm{k}(\mathrm{i})$ represents the absorption coefficient of a chemical substance of corresponding wavelength.

Another important parameter for describing the leaf morphology is the structural parameter $\mathrm{N}$ of the leaf. The nearinfrared high reflectivity of leaves is caused by multiple scattering of cell walls inside and air gaps. It has been shown that when there are many cell layers, the spectral reflectivity of leaves is high; when the anisotropy of cell shape and composition is obvious, the spectral reflectivity is high. In the wavelength with large absorption coefficient, the structural parameter $\mathrm{N}$ has less effects on the leaf spectrum.

For broad-leaved leaves, the calculation of structural parameter is quite complex and it is usually obtained with a empirical formula approximately. The formula is:

$$
\mathrm{N}=\frac{0.9 S L A+0.025}{S L A-0.1}
$$

Where SLA represents the specific leaf area, namely the leaf area per unit dry weight.

From the calculation formula of broad leaves, the specific leaf area is a parameter applicable to the determination and calculation of broad leaves, so obviously it is not appropriate to use the above formula to calculate the structural parameter of coniferous leaves and it is also required to define a structure parameter for broad leaves to represent the structural form of coniferous leaves.

In the generalized model established in this research, the structural parameter of broad leaves and coniferous leaves is believed to characterize the layering mode of mesophyll cells and to affect the diameter of mesophyll cells and the thickness of air gap meantime, so the structural parameter at this time can be regarded as the layer number of cells. For coniferous vegetation, under the circumstance where the cell dimension and leaf thickness are known (generally the cell dimension and leaf thickness of coniferous vegetation are easy to obtain), the measured leaf thickness can be used to determine the leaf-layer structure as the structural parameter in the generalized model. In the model, the structural parameter $\mathrm{N}$ is believed to the layer number of finite cell layer. While, during the application, the structural parameter $\mathrm{N}$ is not required to be an integer, and the calculate result can be used in the model directly. The calculation formula is:

$$
\mathrm{N}=\frac{h+x_{n}}{h-d}
$$

Where $\mathrm{h}$ represents the leaf thickness.

Formula (34) is the formula for calculating the structure parameter of coniferous leaves. For broad-leaved vegetation, the wide variety of vegetation results in that the mesophyll cells of leaves and the gap dimension of cells are difficult to obtain(Benford,1946). Although the two leaf parameters are not used in the PROSPECT model, actually the two are important indexes of describing the physiological and ecological structure of leaves. In this research, the two parameters of broad-leaved vegetation are taken as input variables to establish the generalized model, which is closer to the actual situation. When the model is forward, the broad-leaved vegetation can be regarded as non-compact initially. At this time, the two parameters are deemed to be equal, and the leaf thickness and structural parameter are used to calculate approximate value of the two. The calculation formula is:

$$
\mathrm{d}=\frac{h}{2 * N-1}
$$

Formula (35) is the approximation equation for leaf cell diameter and cell gap. Adjustment can also be done according to the actual leaf structure after the approximation value is obtained.

\section{VALIDATION DATA}

In order to test the simulation precision of the GLIBERTY generalized model, the Chang-chang Expressway from Changesha to Yiyang is selected as a research area in this research. The vegetation coverage in the research area is good and the vegetation consists of broad-leaved forest mainly accompanied with parts of mixed frost. The main tree species is pine accompanied with small cypress. The data collected from filed experiments include physical and chemical parameters and corresponding spectral information of 165 vegetations in total within 36 quadrats. The statistics of leaf parameters after treatment is shown in Table 1.

\begin{tabular}{|l|l|l|l|l|}
\hline Type & \multicolumn{2}{|l|}{ Broad leaf } & \multicolumn{2}{l|}{ Coniferous leaf } \\
\cline { 2 - 5 } Parameter & MAX & MIN & MAX & MIN \\
\hline $\begin{array}{l}\text { Chlorophyll } \\
\text { (mg/g) }\end{array}$ & 61.1 & 29.9 & 42.8 & 34.7 \\
$\begin{array}{l}\text { Leaf water } \\
\text { content(g/cm2) }\end{array}$ & 0.0174 & 0.0027 & 0.163 & 0.123 \\
$\begin{array}{l}\text { Dry matter } \\
\text { content (g/cm2) }\end{array}$ & 0.0519 & 0.0083 & 0.066 & 0.032 \\
$\begin{array}{l}\text { Structural } \\
\text { parameter N } \\
\text { Cell diameter } \\
\text { (mm) }\end{array}$ & 1.7 & 1 & 0.9 & 1.5 \\
$\begin{array}{l}\text { Cell gap (mm) } \\
\text { Leaf thickness } \\
\text { (mm) }\end{array}$ & 0.8 & 0.3 & 0.1 & 0.02 \\
\hline
\end{tabular}

Table 1: Statistical data table

The leaf spectral reflectivity is simulated according to the vegetation parameters and the RMSE is calculated by taking the actual spectrum as standard. The result shows that: for broad leaves, the maximum RMSE is 0.23055 and the minimum RMSE is 0.13825 ; for coniferous leaves, the maximum RMSE is 0.15816 and the minimum RMSE is 0.11842 . The calculation result shows that square root error of the simulation of leaf reflectivity with the generalized model is small.

\section{SENSITIVITY ANALYSIS}

Compared with the broad-leaved model and the coniferous model, the input parameters of the generalized model increases, which contributes to the analysis to the physiological and chemical forms of leaves in the model of leaf optical characteristics and the actual application of the model. Now, sensitivity analysis is done to the two relatively important physical parameters in the model(Fig. 3 and Fig. 4). 


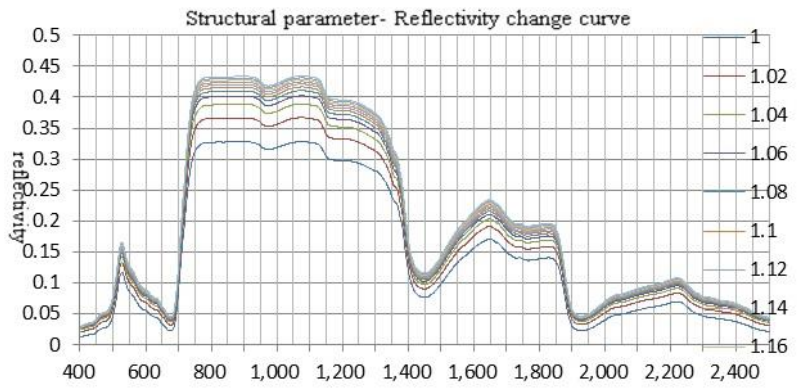

Figure 3. Reflectivity change curve of structural parameter

Firstly, the leaf structural paramerter in the model is analyzed and the change scope of the structural parameter is set to be 1 1.2 with interval of 0.02 . The structural parameter change curve shows that the slight change of the structural parameter will cause significant change of leaf spectrum in the near-infrared region and the sensitivity of near-infrared part to the leaf structural parameter is high. When the structural parameter increases, the spectural reflectivity of leaves will increses too (specially in the near-infrared region) and the reflctivity increasment decreases step by step. Because GLIBERTY considers that the leaf structural parameter characterizses the cell layers in leaves, so the change processs complies with the theory that the reflectivity meased on the cell layer increases along with the increase of layer of cells and the therory that the reflectivity measued tends to stablize when the layer of cells increases to a certain amount. Therefore, the accuracy of the structural parameter shall be noted during the application of the model.

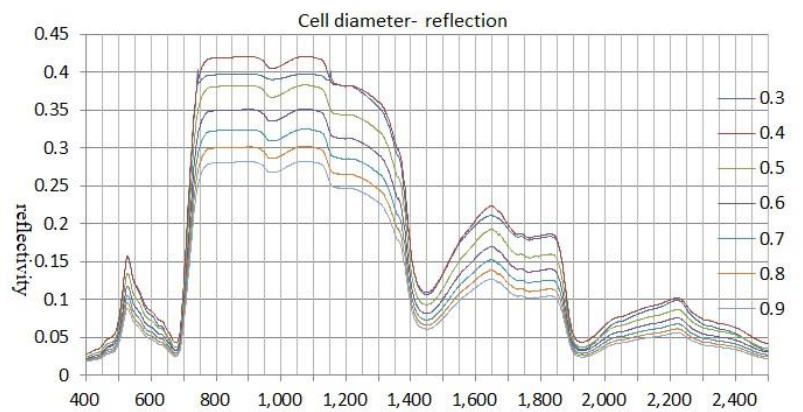

Figure 4. Reflectivity change curve of cell diameter

The cell diameter- reflectivity change curve shows that when the cell diamter increase gradually, the reflectivity of leavse in the short-wave infrared band decreses gradually; when other parameters are fixed, the cell dimension affects the distribution structure of leaf internal cells and causes the change of reflcivity in the near infrared part; meantime, the cell fluid in cells is the main water storage structure of leaves. The change in the curve also shows that the change of cell diamter affects the reflectivity of short-wave infrared part, especially the three absorption vallyes under the effect of water. The analysis shows that the lager the cell dimater, the more the water content, so the water absorption vally decreases. This process reflects the effect on leaf spectrum in the short-wave infrared part from water content from the respective of cell diameter indirectly.

\section{RESULTS AND DISCUSSION}

The generalized model provides a feasible scheme applicable to simulate the leaf optical information for broad leaves and coniferous leaves. The test result shows that:

under the circumstance where only the chlorophyll, equivalent water thickness and dry matter concentration are used for broad-leaved vegetation, the broad-optical curve simulated with cell parameters with lower accuracy is still close to the measured value; under the circumstance where only the chlorophyll, equivalent water thickness and dry matter concentration rather than the basic absorption and other chemical parameters are considered, the spectrum curve simulated with accurate cell parameters can reflect the actual spectrum information of coniferous vegetation better and the gradual application of the generalized model in the vegetation information will provide data support with high accuracy to quantitative remote sensing. The research result shows that the generalized model GLIBERTY applies to broad-leaved vegetation and coniferous vegetation better. During the test, it is found that different models have different sensitivities to input parameters and it is required to study and analyze the sensitivity of models to physical and chemical parameters of vegetation further to determine the accurate model parameter required to input, and to extract model parameters for inversion of vegetation information to improve the precision and practicability of the model; In addition, the change of leaf thickness is related to the vegetation health, so the proposed generalized model provides a new and effective tool for the research on the vegetation health in large area.

\section{REFERENCES}

Bacour, C., \& Baret, F., \& Béal, D. \& Weiss, M., \& Pavageau, K., 2006. Neural network estimation of LAI, fAPAR, fCover and LAI $\times$ Cab, from top of canopy MERIS reflectance data: Principles and validation. Remote Sensing of Environment, 105(4), pp.313-325

Benford, F., 1946. Radiation in a diffusing medium. Journal of the Optical Society of Ameriva, 36(9),pp.524-537

Danson, F. M., \& Aldakheel, Y. Y., 2000. Diurnal water stress in sugar beet: Spectral reflectance measurements and modelling. Agronomie, 20(1),pp.31-391

George, G., \& Stokes.,1860. On the intensity of the light reflected from or transmited through a pile of plates. Proceedings of the Royal Society of London,pp. 545-556

Jacquemoud, S., \& Bacour, C., \& Poilve, H., \& Frang, J. P., 2000. Comparison of Four Radiative Transfer Models to Simulate Plant Canopies Reflectance: Direct and Inverse Mode. REMOTE SENS. ENVIRON, 74(3),pp.471-481

Judd, D. B., 1942. Fresnel reflection of diffusely incident light. Journal of Research of the National Bureau of Standards, 29(6),pp.329-332

Kuusk, A., 1991. Determination of vegetation canopy parameters from optical measurements. Remote Sens. Environ, 37(3),pp.207-218

Laurent Bousquet., \& Sophie Lachéradea.,\& Stéphane Jacquemoud., \& Ismaël 
Moyab., 2005. Leaf BRDF measurement and model for specular and diffuse component differentiation. Remote Sensing of Environment, 98(2),pp.201-211

L. B. TUCKERMAN., 1947. On the intensity of the light Reflected from or Transmitted through a pile of Plates. Journal Of The Optical Soceety Of America,37(10),pp.818

Jacquemoud S, Baret F., 1990. PROSPECT: a model of leaf optical properties spectra.[J]. Remote Sensing of Environment, 34(2),pp.75-91.

Melamed, M. T., 1963. Optical properties of powders. Part I. Optical absorption coefficients and the absolute value of the diffuse reflectance. Journal of Applied Physics ,34(3),pp.560570

Raymond F. Kokaly., \& Gregory P. Asner., \& Scott V. Ollinger., \& Mary E. Martin.,

\& Carol A. Wessman., 2009. Characterizing canopy biochemistry from imaging

spectroscopy and its application to ecosystem studies. Remote Sensing of

Environment, 113(1),pp.78-91

Stern, F., 1964. Transmission of isotropic radiation across an interface between two dielectrics. Applied Optics, 3(1),pp. 111113

Terence, P. D., \& Paul, J. Curran., \& Stephen, E. P., 1998. LIBERTY-Modeling the effects of leaf biochemical concentration on reflectance spectra. Remote Sens. Environ, 65(1),pp.50-60

Verhoef, W., \& Bach, H., 2003. Remote sensing data assimilation using coupled radiative transfer models. Physics and Chemistry of the Earth,28(1-3),pp.3-13

Yang, Y., \& Ling, P. P., 2004. Improved model inversion procedure for plant water status assessment under artificial lighting using PROSPECT+SAIL. Transactions of the ASAE, 47(5), pp.1833-1840

Z. MALENOVSKY., \& J. ALBRECHTOVA., \& Z. LHOTAKOVA., \& R. ZURITA-MILLA., \& J. G. P. W. CLEVERS., \& M. E. SCHAEPMAN., \& P. CUDLIN., 2006. Applicability of the PROSPECT model for Norway spruce needles. International Journal of Remote Sensing, 27(24),pp. 5315-5340 